\title{
The estimation of genetic correlations from phenotypic correlations: a test of Cheverud's conjecture
}

\author{
DEREK A. ROFF \\ Department of Biology, McGill University, 1205 Dr Penfield Ave., Montreal, Canada H3A $1 B 1$
}

\begin{abstract}
The estimation of genetic correlations is central to the study of evolutionary change in populations. However, sample sizes required to achieve a small standard error are typically enormous. This precludes large-scale comparative analyses. Cheverud has conjectured that in some circumstances the phenotypic correlation can be substituted for the genetic correlation. This suggestion is examined using a large set of morphological traits in the sand cricket, Gryllus firmus. In this case the difference between the two estimates is very small. Further, by simulation it is shown that the phenotypic correlations are as good as, or better than, the estimated genetic correlations as estimates of the true genetic correlations. Examination of other data sets of morphological traits suggests that the phenotypic correlation may, in general, be a suitable substitute for the estimated genetic correlation. However, because the number of such examinations is still small, a protocol is suggested in which two sets of genetic analyses are undertaken to confirm the assumption in a large comparative analysis.
\end{abstract}

Keywords: bias, evolution, genetic correlation, Pearson product-moment correlation, phenotypic correlation.

\section{Introduction}

The quantitative analysis of the evolution of a suite of traits requires two sets of parameters, the heritabilities of the traits $\left(h^{2}\right)$ and the correlations between each pair. These correlations are made up of two elements, the genetic correlation $\left(r_{\mathrm{G}}\right)$, which is the correlation of breeding values, and the environmental correlation $\left(r_{E}\right)$, which is the correlation of environmental deviations plus nonadditive genetic deviations (Falconer, 1989). Unfortunately, the estimation of the genetic correlation requires extremely large sample sizes (Klein et al., 1973). For example, suppose for two traits $x, y, h_{x}^{2}=h_{y}^{2}=h^{2}$, and we utilize a full-sib breeding design with a total sample size of $T$ individuals. Utilizing equations 10.14 and 19.4 of Falconer (1989, p. 183 and p. 317, respectively) an approximate estimate of the standard error of the genetic correlation in this case is

$\mathrm{SE}=\left(1-r_{\mathrm{G}}^{2}\right) \sqrt{\frac{8}{T h^{2}}}$.

For morphological traits a typical heritability is 0.4 (Mousseau \& Roff, 1987): for $r_{\mathrm{G}}=0.5$, and $T=100$, the standard error is 0.34 , which because of the wide confidence interval makes the estimate of little value. Increasing $T$ by an order of magnitude to 1000 decreases the standard error to 0.11 , which still means a wide confidence interval. Thus large-scale comparisons of genetic correlations among populations or among species are generally impractical.

The phenotypic correlation $\left(r_{\mathrm{P}}\right)$, which is simply the standard Pearson product-moment correlation between the two phenotypic values of the traits, is more easily measured than either the genetic or environmental correlations, and is a simple function of the two: $r_{\mathrm{P}}=r_{\mathrm{G}} \sqrt{h_{x}^{2} h_{y}^{2}}+r_{\mathrm{E}} \sqrt{\left(1-h_{x}^{2}\right)\left(1-h_{y}^{2}\right)}$.

The standard error of the phenotypic correlation is approximately $1 / \mathrm{J}(T-3)$ (Sokal \& Rohlf, 1981: in practice correlations are transformed to the $z$-scale, but this makes little difference in the present case): the standard errors for the two cases considered above are 0.10 and 0.03 , respectively. Thus for the same sample size the standard error is reduced threefold. Clearly, if we could substitute the phenotypic for the genetic correlation we could considerably reduce experimental effort. Further, the phenotypic correlation can be obtained far more easily than the genetic correlation, since restrictions on mating design are much less. 
Based on an analysis of 41 pairs of phenotypic/ genetic correlation matrices Cheverud (1988, p. 958) concluded that 'phenotypic correlations are likely to be fair estimates of their genetic counterparts in many situations'. The analysis of Roff \& Mousseau (1987) on the phenotypic and genetic correlations in Drosophila suggested that the former might be reasonable estimates of the latter when only morphological traits are considered. In this regard it is noteworthy, as pointed out by Cheverud, that almost all of the traits considered in his analysis were morphological traits (Cheverud, 1988). Koots \& Gibson (1994) also found in a survey of traits related to beef production a very high correlation between the genetic and phenotypic correlations.

In this paper I examine Cheverud's conjecture for morphological traits in the sand cricket, Gryllus firmus. Using these data I construct a simulation model to examine the circumstances under which the phenotypic correlations will be a better measure of the genetic correlations than the estimates of the genetic correlations themselves. Finally, I survey the literature for data from other nondomestic species in which reasonably large numbers of phenotypic and genetic correlations are reported.

\section{Materials and methods}

\section{Experimental protocol}

Gryllus firmus is a wing-dimorphic, ground-dwelling cricket inhabiting sandy sites along the eastern seaboard of the USA (Alexander, 1968; Harrison, 1985). From approximately 40 individuals collected in northern Florida a continuously breeding laboratory stock was established. Heritabilities and genetic correlations were estimated using full-sib families drawn from this stock, the parents being raised at $28{ }^{\circ} \mathrm{C}$ and a photoperiod of 15L:9D. Each family consisted of 120 newly hatched nymphs divided between two $4 \mathrm{~L}$ buckets. Food, in the form of rabbit chow, was provided ad libitum and water was supplied via a cheesecloth wick that passed through the bottom of the bucket into a reservoir beneath. Two experiments were undertaken:

1 Environment 1: 49 families raised at $25^{\circ} \mathrm{C} 15 \mathrm{~L}: 9 \mathrm{D}$, conditions that favour the production of micropterous (short-winged, flightless) adults, and

2 Environment 2: 44 families raised at $30^{\circ} \mathrm{C} 17 \mathrm{~L}: 7 \mathrm{D}$, conditions that promote the production of macropterous adults (long-winged, volant).

To avoid the confounding problems associated with variation related to morph, in the first experiment only macropterous offspring were measured, and in the second experiment only micropterous offspring were measured. Because rearing conditions varied between the two experiments it is not possible to discriminate effects arising from rearing conditions from effects related to morph. This is not, however, an issue in the present analysis since the object of the analysis is to compare phenotypic and genetic correlations within experiments only.

Adults were collected at final moult and kept for 1 week in single-sex groups to permit development of the gonads, after which they were preserved in Bouin's fluid. I attempted to collect a minimum of 10 individuals of each sex from each family, with equal numbers from each bucket. Though survival was high ( 74 per cent for experiment 1, and 71 per cent for experiment 2 ), this was not always possible because of insufficient numbers of the appropriate wing morph (mean family sizes were: Experiment 1, $n=9.7$ for males, $n=12.0$ for females; Experiment 2, $n=12.8$ for males, $n=8.9$ for females). A total of 12 morphological measurements were taken from the females and 11 from the males: total weight, head weight, thorax weight, abdomen weight, gonad weight, head width, thorax length, front thorax width, rear thorax width, femur length, wing length, and ovipositor length (females only).

\section{Statistical analysis}

Full-sib estimates of heritabilities and genetic correlations are potentially biased by dominance components and common environment effects. The latter were accounted for by the use of a nested ANOvA (cages nested within family). Heritability estimates from fullsib analysis may include one quarter of the dominance variance (Falconer, 1989), although comparison between full-sib estimates and estimates from offspring-parent regression indicates that, in animals in general, the full-sib method does not overestimate narrow sense heritability (Mousseau \& Roff, 1987). Further, full-sib estimates for wing morph (Roff, 1986), development time (Roff, 1990) and head width (Roff, unpublished data) in $G$. firmus are not significantly different from offspring-parent regression estimates.

Except when the data set is fully balanced the statistical properties of the estimates of heritability and genetic correlation are not known (Becker, 1985). Simulation modelling has shown that reliable estimates of the parameters and their associated standard errors can be obtained by jackknifing the estimates obtained using the variance components from the ANOVA (Simons \& Roff, 1994; Roff \& Preziosi, 1994). This technique was used in the present analysis.

The phenotypic and genetic correlations were compared in two ways. First, the genetic correlation was regressed on the phenotypic correlation. Because the 
data clearly violate the assumptions of linear regression, the probability level was assessed using Mantel's test (Cheverud, 1988). With respect to Cheverud's conjecture we require that the estimates cluster closely around the line of equality. A trend to deviate from this line may indicate a systematic difference between the two estimates; however, statistical testing of such a trend is difficult because the estimates are not independent, and hence the results from the parametric analysis must be viewed with caution. A systematic bias may not be important if it is small; indeed a small bias with the points nevertheless lying close to the $1: 1$ line is preferable to one in which there is no bias but in which the points are scattered very widely about the 1:1 line. To assess how close, on average, were the two estimates I used the mean absolute difference, $D=\Sigma\left|r_{\mathrm{G}, i, j}-r_{\mathrm{P}, i, j}\right| / n$, where $i, j$ refer to characters $i$ and $j(i \neq j)$ and $n$ is the total number of correlations. Correlations involving total weight and the weight of some other body component were not used as they cannot in principle be uncorrelated (there are four such correlations). The total number of correlations for females was thus 62 , and for males the total number was 51.

\section{Simulation modelling}

It is difficult to construct a general model that simulates the type of complex phenotypic and genetic relationships examined here (for a model that simulates two characters see Roff \& Preziosi, 1994). However, it is possible to construct a specific model by making use of the observed data set (Mueller, 1979). In this approach the data set is used as a complete descriptor of the actual population, and thus the 'true' correlations are those obtained in the original data set. Samples are drawn at random with replacement, thereby generating simulated data sets. Two types of sampling schemes were employed: first, the sample sizes in the simulated data sets exactly matched those of the original data set (i.e. if family $k$ contained $n_{k}$ individuals then $n_{k}$ individuals were sampled from this family), and secondly, the sample sizes were 2 and 3 individuals per cage. From each simulated set two statistics were calculated,

$D_{\mathrm{G}}=\frac{\sum_{i=1}^{i=n}\left|R_{\mathrm{G}, i}-r_{\mathrm{G}, i}\right|}{n}$
$D_{\mathrm{P}}=\frac{\sum_{i=1}^{i=n}\left|R_{\mathrm{G}, i}-r_{\mathrm{P}, i}\right|}{n}$,

where $R_{\mathrm{G}, i}$ is the 'true' $i$ th genetic correlation (i.e. those obtained from the original data set), $r_{\mathrm{G}, i}$ and $r_{\mathrm{P}, i}$ are the estimated genetic and phenotypic correlations obtained from the simulated data set, and $n$ is the total number of correlations ( 62 for females, 51 for males). Five hundred simulated data sets were constructed for each combination of experiment and sex for the first sampling scheme, and using the female data from experiment 1 for the second sampling scheme. Using the resulting distributions of $D_{\mathrm{G}}$ and $D_{\mathrm{P}}$, three questions were addressed:

1 what is the probability of obtaining a data set in which the mean absolute difference between the phenotypic correlation and the true genetic correlation is smaller than that between the true and estimated genetic correlations (i.e. probability of $D_{\mathrm{P}}<D_{\mathrm{G}}$ )?

2 what is the mean difference between $D_{\mathrm{G}}$ and $D_{\mathrm{P}}$ ?

3 what are the relative variabilities of $D_{\mathrm{G}}$ and $D_{\mathrm{P}}$ ?

An estimator that has a small bias but small variance may be preferred over estimates that have a high probability of being markedly different from the true values. Therefore, if the average difference between $D_{\mathrm{G}}$ and $D_{\mathrm{P}}$ is small (question 2), the probability of $D_{\mathrm{p}}<D_{\mathrm{G}}$ high (question 1), and the variability in $D_{\mathrm{G}}$ much greater than that in $D_{\mathrm{p}}$ (question 3 ), then the phenotypic correlations may be acceptable, or even preferred, estimates of the genetic correlations.

\section{Results}

\section{Experimental results}

Males and females differed significantly in the mean values of all traits and therefore analyses were done separately for each sex. Heritabilities varied from 0.19 to 0.84 , but the majority ( 89 per cent) fell within the range $0.35-0.55$ (Table 1 ), which is consistent with the heritabilities of morphological characters in general (Mousseau \& Roff, 1987). In all cases there was a highly significant correlation between the genetic and phenotypic correlations $(P<0.0001$, both from the parametric and randomization tests, Fig. 1 and Table 2 ). For the males in environment 1 the genetic correlations were consistently smaller than the phenotypic correlations, but in environment 2 the genetic correlations were consistently larger (Fig. 1). For the females in the first environment more correlations lay below the 1:1 line but this was not significant ( 38 of 62 , $\chi_{1}^{2}=3.2, P>0.05$, while in the second environment the slope did not differ from 1 nor did the intercept differ from zero, but significantly more genetic correlations lay above the 1:1 line than expected by chance ( 46 of $62, \chi_{1}^{2}=14.5, P<0.001, \chi^{2}$ goodness of fit). For both males and females the mean absolute difference between the genotypic and phenotypic correlations was small, ranging from 0.08 to 0.12 (Table 2). 
Table 1 Heritability estimates for Gryllus firmus

\begin{tabular}{|c|c|c|c|c|}
\hline \multirow[b]{2}{*}{ Character } & \multicolumn{2}{|c|}{ Female } & \multicolumn{2}{|c|}{ Male } \\
\hline & $h^{2}$ & SE & $h^{2}$ & SE \\
\hline \multicolumn{5}{|l|}{ Environment 1} \\
\hline Total weight & 0.43 & 0.10 & 0.39 & 0.12 \\
\hline Head weight & 0.40 & 0.09 & 0.48 & 0.10 \\
\hline Thorax weight & 0.44 & 0.10 & 0.19 & 0.11 \\
\hline Abdomen weight & 0.43 & 0.10 & 0.33 & 0.12 \\
\hline Gonad weight & 0.49 & 0.11 & 0.47 & 0.14 \\
\hline Head width & 0.36 & 0.11 & 0.35 & 0.11 \\
\hline Thorax length & 0.35 & 0.09 & 0.57 & 0.09 \\
\hline Front thorax width & 0.35 & 0.10 & 0.36 & 0.10 \\
\hline Rear thorax width & 0.41 & 0.09 & 0.32 & 0.09 \\
\hline Femur length & 0.30 & 0.11 & 0.38 & 0.11 \\
\hline Wing length & 0.42 & 0.10 & 0.27 & 0.09 \\
\hline Ovipositor length & 0.31 & 0.09 & - & - \\
\hline \multicolumn{5}{|l|}{ Environment 2} \\
\hline Total weight & 0.40 & 0.16 & 0.53 & 0.16 \\
\hline Head weight & 0.39 & 0.14 & 0.55 & 0.15 \\
\hline Thorax weight & 0.33 & 0.13 & 0.63 & 0.17 \\
\hline Abdomen weight & 0.43 & 0.15 & 0.55 & 0.14 \\
\hline Gonad weight & 0.28 & 0.15 & 0.84 & 0.16 \\
\hline Head width & 0.41 & 0.13 & 0.42 & 0.14 \\
\hline Thorax length & 0.22 & 0.17 & 0.52 & 0.13 \\
\hline Front thorax width & 0.42 & 0.14 & 0.47 & 0.14 \\
\hline Rear thorax width & 0.51 & 0.15 & 0.63 & 0.15 \\
\hline Femur length & 0.33 & 0.16 & 0.48 & 0.11 \\
\hline Wing length & 0.38 & 0.12 & 0.49 & 0.13 \\
\hline Ovipositor length & 0.39 & 0.13 & - & - \\
\hline
\end{tabular}

It is evident from the approximate formula for the standard error of the genetic correlation presented in the Introduction that the standard error increases as the genetic correlation decreases (i.e. for fixed heritabilities $\left.\mathrm{SE} \propto 1-r_{\mathrm{G}}{ }^{2}\right)$. The increase in $\mathrm{SE}$ is very marked, and consequently many genetic correlation estimates have confidence intervals that include zero (Fig. 2). There is also an increase in the standard error of the phenotypic correlation as this correlation decreases, but the change is relatively slight (Fig. 2).

\section{Simulation results}

1. Sample sizes same as in original data. The probability that the phenotypic correlation lies closer to the true genetic correlation than the estimated genetic correlation ranged from 0.08 to 0.44 ('Probability $D_{\mathrm{P}}<D_{\mathrm{G}}$ ' in Table 3 ). However, there was virtually no difference between the mean $D_{\mathrm{G}}$ and the mean $D_{\mathrm{p}}$, the maximum difference between the two being 0.03 . Thus if the true genetic and phenotypic correlations are as observed in the data sets, use of the phenotypic correlations as estimates of the genetic correlations will introduce negligible bias. The variances of $D_{\mathrm{G}}$ were considerably larger than those of $D_{\mathrm{P}}$ (Table 3, Fig. 3), and consequently in any given sample the estimated genetic correlations could be enormously different from the true genetic correlations. Therefore, in these two data sets the phenotypic correlations are at least as good as estimators of the genetic correlations as are the estimated genetic correlations.

2. Sample sizes of 2 and 3 per cage. Reducing the sample size per family had virtually no effect on the phenotypic correlations but dramatically increased the mean absolute difference between the true and the estimated genetic correlations (Table 3, Fig. 4). With both sample sizes the mean absolute difference of the phenotypic correlations was more likely to lie closer to the true genetic correlation than the estimated genetic correlation itself ( 0.87 and 0.67 , respectively). Thus, in these cases, the phenotypic correlations are superior estimates of the true genetic correlations.

\section{Discussion}

With respect to morphological traits in G. firmus and the sample sizes investigated, the phenotypic correlations are at least as good as estimators of the genetic correlations as are the estimated genetic correlations themselves. As demonstrated by the simulation model, for smaller sample sizes than used in the present experiments the phenotypic correlations are most likely to be superior estimates. How frequently this will be true will, of course, depend upon the relationship between the phenotypic and genetic correlations. Further simulation modelling is required to establish the range of conditions under which phenotypic correlations are better estimates. However, in the final analysis the problem is largely empirical, and we need more studies in which the sample sizes are sufficiently large to determine statistically the pattern between the two types of correlations.

Willis et al. (1991) raised the following four objections to the study by Cheverud (1988).

\section{Objection 1}

'Nearly all of the data (at least 36 out of the 41 studies) are based on populations reared under laboratory or agricultural conditions' (Willis et al., 1991, p. 441). Because of increased environmental variation and possible genotype-by-environment interaction, Willis et al. (1991) suggested that the heritabilities and genetic 
Fig. 1 Genetic vs. phenotyic correlations among male and female Gryllus firmus reared in two environments. Solid line, $1: 1$ relationship.
Males
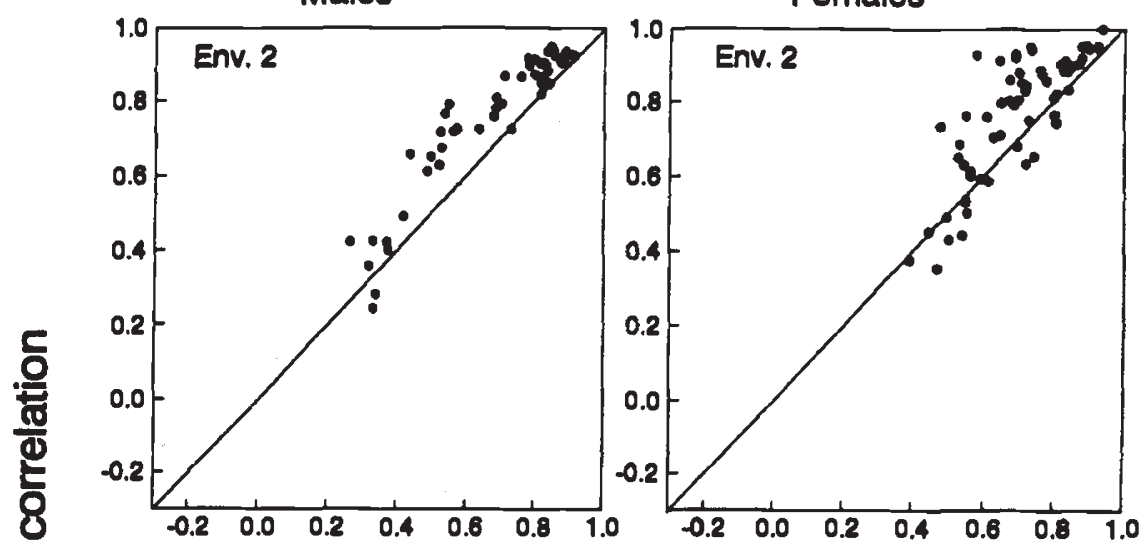

ํㅜㄹ
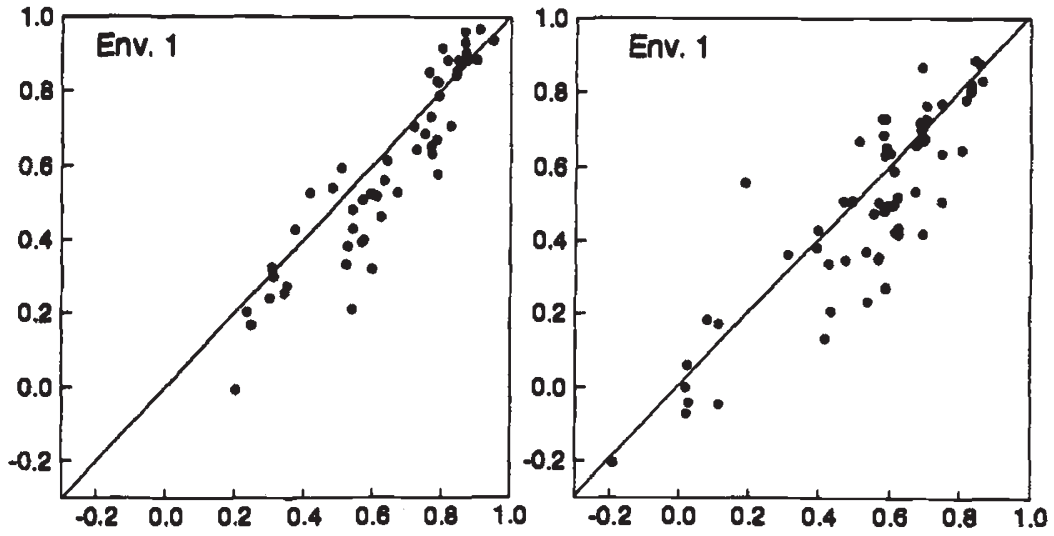

Phenotypic correlation

Table 2 Parameters for the regression of genetic correlation on phenotypic correlation, and the mean absolute difference $(D)$

\begin{tabular}{|c|c|c|c|c|c|}
\hline Environment & Sex & Slope & Intercept & $r$ & $D$ \\
\hline 1 & q & $0.93^{*}$ & -0.01 & 0.86 & 0.12 \\
\hline 1 & 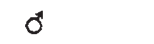 & $1.14^{*}$ & $-0.14 \ddagger$ & 0.92 & 0.09 \\
\hline 2 & \% & $0.99^{*}$ & 0.08 & 0.81 & 0.12 \\
\hline 2 & 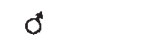 & $0.93^{*}$ & $0.13 \ddagger$ & 0.94 & 0.08 \\
\hline \multicolumn{6}{|c|}{ Results from other studies } \\
\hline \multicolumn{2}{|c|}{ Gryllus pennsylvanicus (lab.), 웅 } & $0.70 \dagger$ & $0.28 \ddagger$ & 0.92 & 0.13 \\
\hline \multicolumn{2}{|c|}{ G. pennsylvanicus (lab.), đ } & $0.95^{*}$ & $0.10 \ddagger$ & 0.99 & 0.07 \\
\hline \multicolumn{2}{|c|}{ G. pennsylvanicus ('field'), ૧ } & $1.20 \dagger$ & $-0.13 \ddagger$ & 0.99 & 0.04 \\
\hline \multicolumn{2}{|c|}{ G. pennsylvanicus ('field'), đ } & $1.39 \dagger$ & $-0.30 \ddagger$ & 0.99 & 0.05 \\
\hline \multicolumn{2}{|c|}{ Geospiza conirostris } & $1.17^{*}$ & -0.10 & 0.62 & 0.13 \\
\hline \multicolumn{2}{|l|}{ G. scandens } & 0.63 & -0.21 & 0.34 & 0.50 \\
\hline \multicolumn{2}{|l|}{ G. fortis } & $1.40 \dagger$ & -0.09 & 0.98 & 0.20 \\
\hline \multicolumn{2}{|c|}{ Melospiza melodia } & $0.76^{*}$ & 0.01 & 0.72 & 0.11 \\
\hline
\end{tabular}

*Significantly different from 0.0 but not from $1.0, \uparrow$ significantly different from 1.0 , $\ddagger$ significantly different from 0.0 .

G. pennsylvanicus: femur length, head width, thorax length, thorax width, ovipositor length (Simons \& Roff, 1995).

G. conirostris: weight, wing length, tarsus length, four bill dimensions (Grant, 1983).

$G$. scandens and $G$. fortis: weight, wing cord, tarsus length, four bill dimensions (Boag, 1983).

M. melodia: wing length, tarsus length, three bill dimensions (Schluter \& Smith, 1986). 


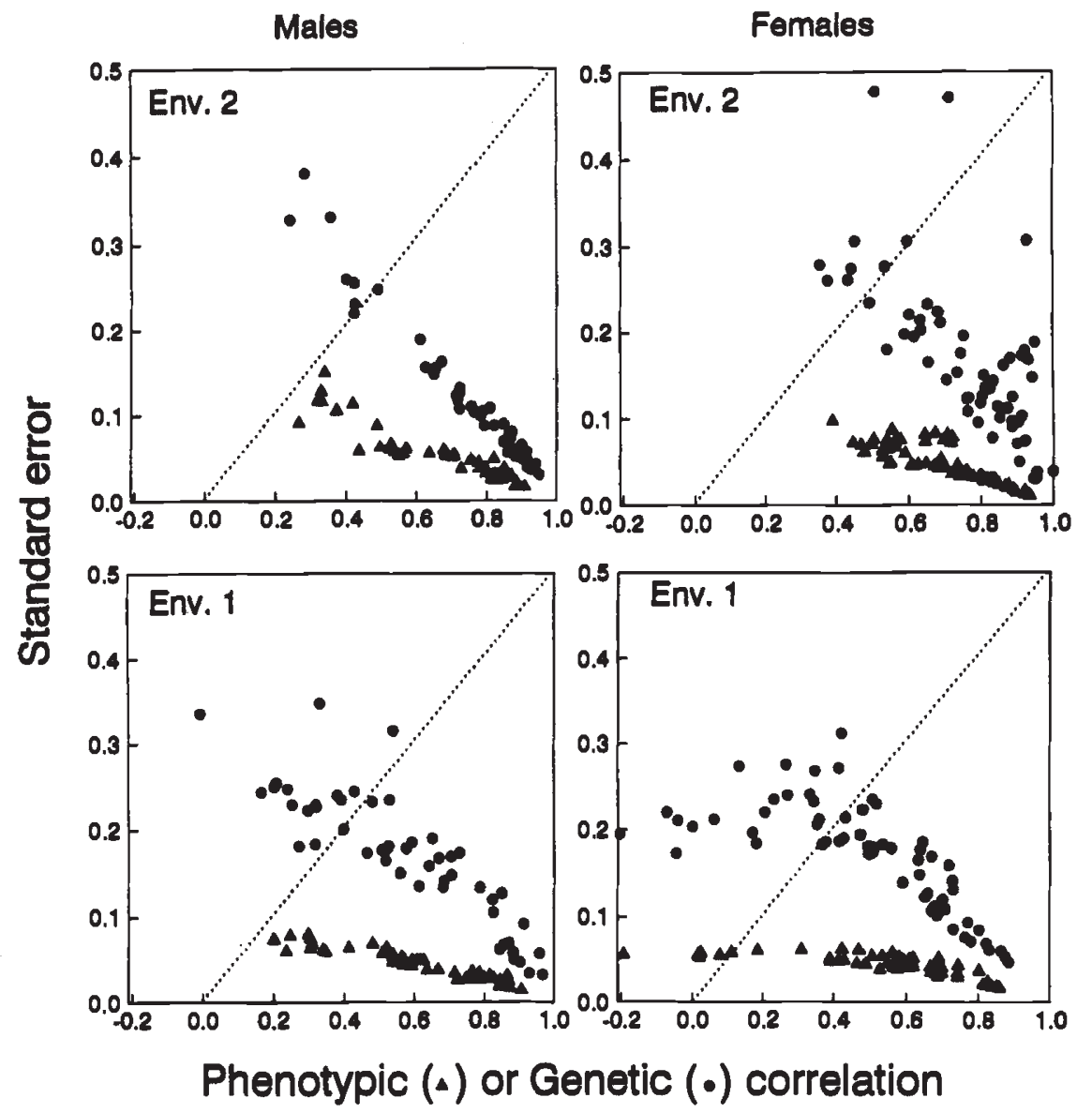

Fig. 2 Standard error of the estimated phenotypic (triangle) and genetic (circle) correlations vs. the estimate. Confidence intervals of estimates lying above the dotted line include zero.

Table 3 Statistics from the simulation analysis

\begin{tabular}{|c|c|c|c|c|c|c|c|c|}
\hline \multirow[b]{2}{*}{ Environment } & \multirow[b]{2}{*}{ Sex } & \multicolumn{2}{|c|}{ Mean } & \multicolumn{2}{|c|}{ Variance } & \multicolumn{2}{|c|}{ Maximum } & \multirow{2}{*}{$\begin{array}{c}\text { Probability } \\
D_{\mathrm{P}}<D_{\mathrm{G}}\end{array}$} \\
\hline & & $D_{\mathrm{G}}$ & $D_{\mathrm{P}}$ & $D_{\mathrm{G}}$ & $D_{\mathrm{P}}$ & $D_{\mathrm{G}}$ & $D_{\mathrm{P}}$ & \\
\hline 1 & q & 0.10 & 0.11 & 0.26 & 0.05 & 0.77 & 0.14 & 0.21 \\
\hline 1 & 0 & 0.11 & 0.10 & 0.51 & 0.09 & 0.93 & 0.13 & 0.44 \\
\hline 2 & q & 0.10 & 0.11 & 0.55 & 0.08 & 1.36 & 0.15 & 0.18 \\
\hline 2 & 0 & 0.05 & 0.08 & 0.03 & 0.02 & 0.18 & 0.13 & 0.08 \\
\hline $3 /$ cage & & 0.16 & 0.12 & 0.50 & 0.01 & 0.75 & 0.17 & 0.67 \\
\hline $2 /$ cage & & 0.21 & 0.13 & 1.66 & 0.03 & 1.43 & 0.19 & 0.87 \\
\hline
\end{tabular}

correlations of field populations will be reduced, making 'the correspondence between $r_{\mathrm{P}}$ and $r_{\mathrm{G}}$ smaller in the field than it is in the laboratory' (p. 441). Despite this long-standing objection to the measurement of heritabilities and genetic correlations in the laboratory there are few empirical data to suggest that this is or is not likely to be a major problem. Average heritabilities of morphological traits in four bird species measured in natural populations are well within the range of heri- tabilities of morphological traits measured in the laboratory (mean $h^{2}$ s are: Melospiza melodia, 0.44; Geospiza scandens, $0.43 ; G$. fortis, $0.88 ; G$. conirostris, 0.93; data from Schluter \& Smith, 1986). The correlations between the genetic and phenotypic correlations, however, show a wide variation (Fig. 5, Table 2). In two cases ( $G$. conirostris, $M$. melodia) the relationship is not significantly different from $1: 1$; in one case ( $G$. fortis) the slope is significantly greater than 1 ; and in one case 
Fig. 3 Frequency plots of $D$ from the simulation employing sampling scheme 1 , in which family sizes are the same as in the original data set. $D_{\mathrm{G}}$, black bars; $D_{\mathrm{p}}$, shaded bars.

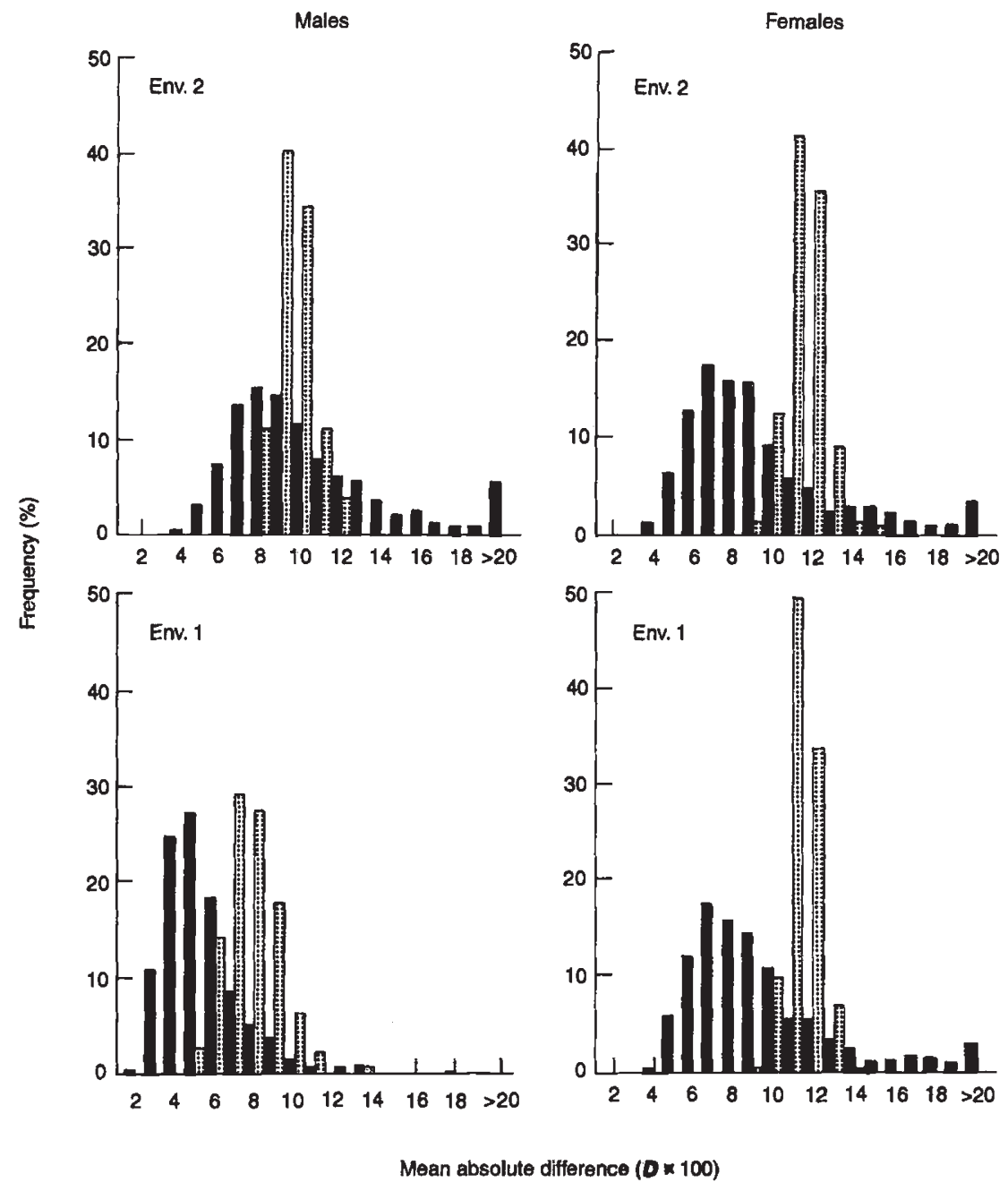

(G. scandens) there is no significant relationship. For the first two cases the mean absolute difference between the phenotypic and genetic correlations, $D$, is very similar to that obtained in the present experiments, while in the others it is larger (Table 2).

All estimates were made using offspring-parent regression, and in two cases the number of families is extremely small ( $n=16$ and 20 for $G$. scandens and $G$. conirostris, respectively), which may account for the nonsignificant relationship observed in $G$. scandens. The negative correlations found in $G$. fortis are all the result of a single morphological measure, LA4, bill length at a depth of $4 \mathrm{~mm}$. This is a rather unusual measure and is clearly correlated in some fashion with other bill measures, such as bill length, of which it is a part. Elimination of this measure from the set produces a significant correlation between genetic and phenotypic correlations $(r=0.70, n=15, P<0.01)$, and now the slope is not significantly different from one but the intercept is significantly greater than zero (i.e. genetic correlations $>$ phenotypic correlations, Fig. 5). Thus, contrary to 'conventional wisdom', the genetic correlations among these four natural populations of birds are not less than the phenotypic correlations, and in one case, $G$. fortis, may be actually greater than the phenotypic correlations.

Simons \& Roff (1995) tested the hypothesis that genetic correlations will be reduced under field conditions by splitting full-sib families of Gryllus pennsylvanicus, a species closely related to $G$. firmus, into two groups and rearing one group under a constant, laboratory environment and the other outside at the site from which the parents had been collected. The crickets reared under the 'field' conditions experienced the same varying photoperiods and temperature fluctuations as the natural population. In both environments there was a highly significant correlation between the genetic and phenotypic correlations, with $D$ s comparable to those found in G. firmus (Table 2). Despite the fact that the relationships may have been statistically 


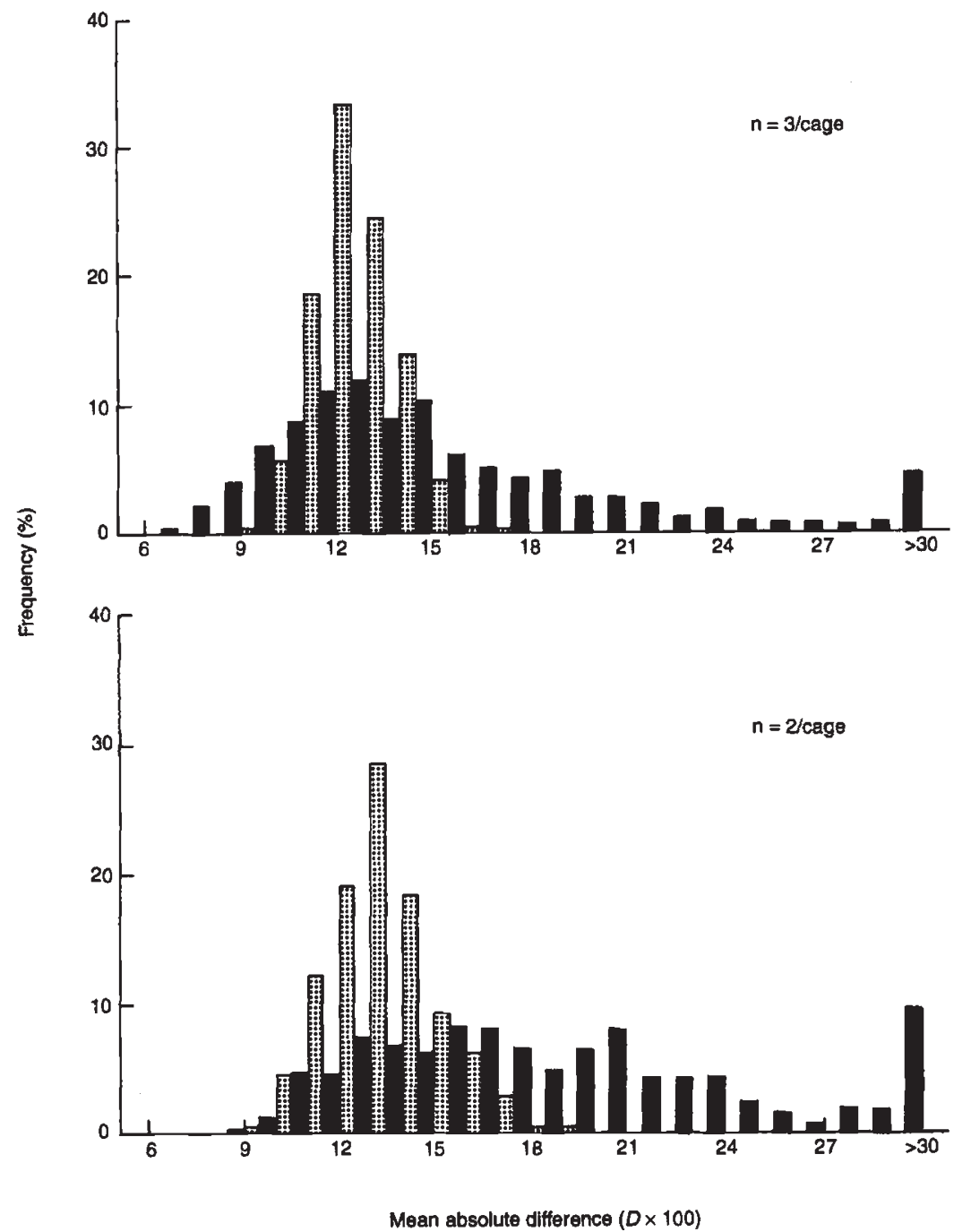

Fig. 4 Frequency plots of $D$ from the simulation employing sampling scheme 2 , in which sample size per cage is kept constant. $D_{\mathrm{G}}$, black bars; $D_{\mathrm{P}}$, shaded bars. different from $1: 1$, the difference between the estimates was so small $(0.04<D<0.13)$ that the phenotypic correlations could easily be substituted for the genetic correlations. Further, the phenotypic and genetic correlations in the laboratory environment were highly correlated with the equivalent correlation in the 'field' environment $(r>0.97)$, and did not differ from a 1:1 relationship (Simons \& Roff, 1995).

Thus, although care should always be taken in extrapolating from the laboratory to the field, the above data suggest that laboratory results are reasonable indicators of the situation likely to be found under natural conditions. Because environmental conditions may alter correlations, it is advisable to conduct experiments, where possible, in several environments.

\section{Objection 2}

Almost all the traits included in the analysis by Cheverud are morphological and may not be relevant to other categories of traits. This was noted by Cheverud as a limitation to his analysis. Examination of genetic and phenotypic correlations among morphological traits in Drosophila suggests that, while the signs of the phenotypic and genetic correlations are generally in the same quadrant (i.e. both positive or both negative), those involving other types of traits (e.g. life history) may not have the same sign (Roff \& Mousseau, 1987). This may be a consequence of difficulties of accurately estimating correlations in nonmorphological traits, but until this is shown one should not use inferences drawn from an analysis of morphological traits alone.

All of the genetic correlations described for $G$. firmus, $G$. pennsylvanicus, and the four bird species were morphological, although in the two cricket species, because of a high correlation between ovary weight and egg number, gonad weight in females might also be classified as a life history trait (Roff, 1994; Simons \& Roff, 1994). Development time was also 
Fig. 5 Genetic vs. phenotypic correlations in four natural populations of birds. Solid line, 1:1 relationship; dotted line, fitted regression.

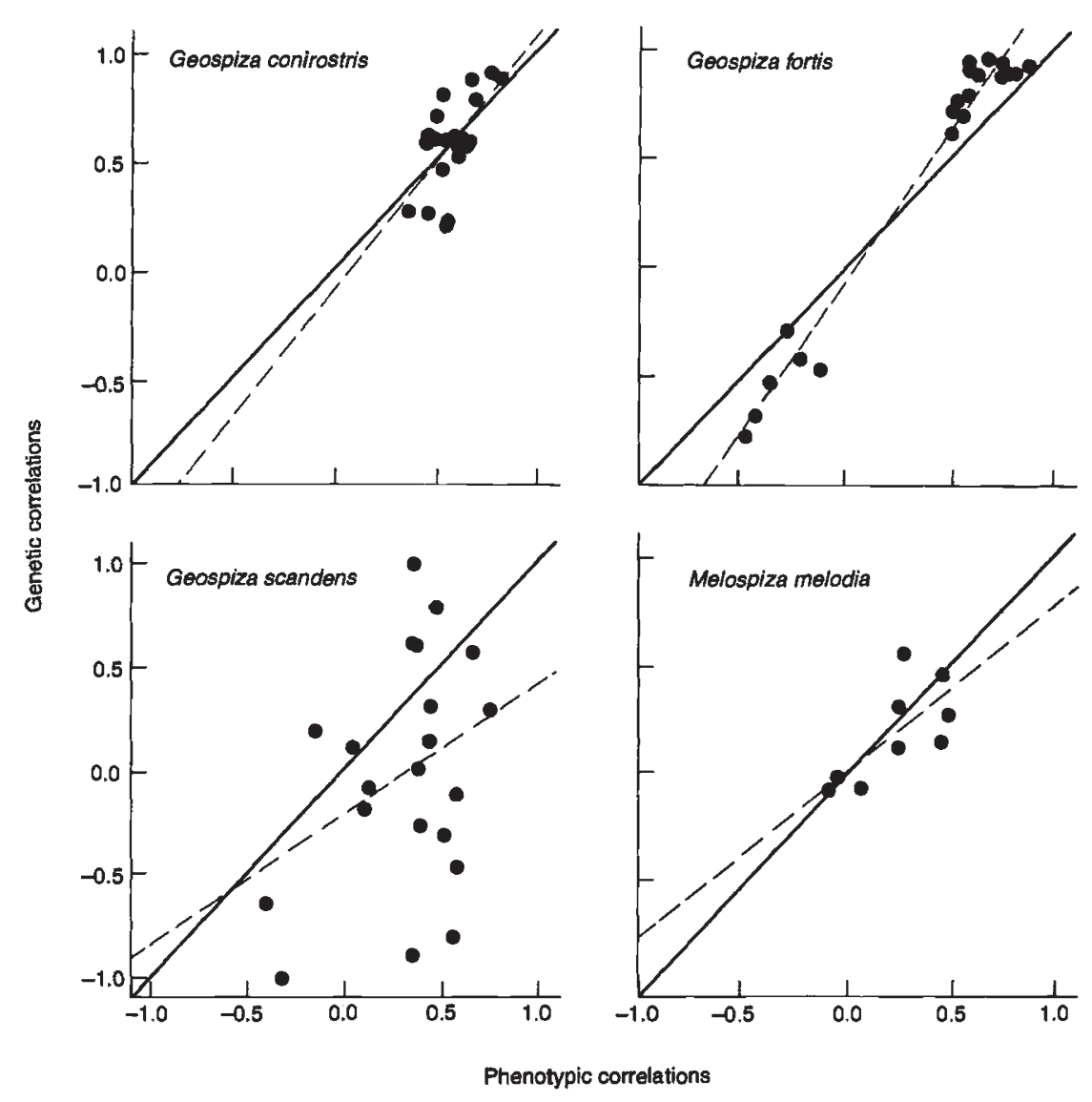

measured in G. pennsylvanicus: in both the laboratory and 'field' environments there were significant correlations between the genetic and phenotypic correlations of development time vs. a morphological trait (lab., $r=0.69, \quad n=9, P=0.039$; 'field', $r=0.67, n=9$, $P=0.049$, males and females combined, data from Simons \& Roff, 1995, morphological traits described in Table 2) and the regression lines did not differ from the 1:1 relationship. These data suggest that in some cases the phenotypic correlations may be reasonable estimates of the genetic correlations for traits other than morphological. However, it must be clearly noted that the results and conclusions given in the present paper refer specifically to morphological traits only.

\section{Objection 3}

Willis et al. (1991) object to the suggestion by Cheverud (1988) that a constant average heritability might be used when data are lacking. While I agree with this objection, it has nothing to do with the use of the phenotypic correlations in place of the genetic, except if one wished to make predictions about the rate of change. In the latter case heritabilities should be estimated directly.

\section{Objection 4}

Cheverud's statistic of disparity between genetic and phenotypic correlations, defined as $\left(\Sigma r_{\mathrm{G}, i, j}^{2}-\Sigma r_{\mathrm{P}, i, j}^{2}\right)$ $/ n$, does not accurately reflect the disparity between the two correlations (Willis et al., 1991). Suppose, for example, that in one case $r_{\mathrm{G}}=r_{\mathrm{P}}=0.2$, and in another, $r_{\mathrm{G}}=-0.2, r_{\mathrm{P}}=0.2$. In both examples Cheverud's statistic gives the same difference, although in the second case there is clearly a discrepancy. However, this statistic was never intended as a statistic of disparity but rather as 'a general measure of intensity of integration (or relationship) in a single matrix as a whole, not as disparity in single coefficients' (Cheverud, personal communication). Nevertheless, I agree with Willis et al. that in order to test the conjecture that the phenotypic correlation can be substituted for the genetic correlation a measure of disparity that takes into account differences in sign is necessary. This has been accomplished in the present paper by using the absolute difference between the correlations: thus, for the example cited above, in the first case, $D=0$, and in the second, $D=0.4$, which does reflect the discrepancy.

More analyses of the relative difference between genetic and phenotypic correlations need to be made 
before, even in the case of morphological traits, we can conclude that, in general, the phenotypic correlations are suitable estimates of the genetic correlations. Nevertheless, the data presented herein are encouraging and suggest the following protocol. Suppose we wish to compare genetic correlations among a range of populations or possibly closely related species (e.g. between populations of $G$. firmus or between species within the genus Gryllus). First, we conduct a detailed genetic analysis, such as undertaken here, for a single species or population. As suggested above, this should be done under several environmental conditions. Because of the potentially confounding influences of dominance effects, a half-sib or offspring-parent regression is preferable to the full-sib design. If the correspondence between genetic and phenotypic correlations is deemed sufficiently high, and this will be a matter of subjective judgement, then we can proceed to compare the phenotypic correlations between populations and species. Using phenotypic correlations will permit many more comparisons than are possible if genetic analyses must be carried out for each population/species. Finally, one or more disparate populations/species should be selected for detailed genetic analysis to confirm further the assumption that phenotypic correlations reflect genetic correlations, within acceptable limits. While this research programme is not a trivial undertaking it would be, at the present time, unwise to proceed in the absence of confirmatory genetic analyses. At the same time the suggested protocol potentially makes large-scale comparisons feasible.

\section{Acknowledgements}

I am most grateful for the constructive comments of Drs J. Cheverud, D. J. Fairbairn, Y. Carrière, I. Weigensberg, and R. Preziosi on earlier drafts of this manuscript. This research was supported by a grant from the Natural Sciences and Engineering Council of Canada.

\section{References}

ALEXANDER, R. D. 1968. Life cycles, specialization and related phenomena in crickets. Q. Rev. Biol., 43, 1-41.

BECKER, w. A. 1985. Manual of Quantitative Genetics. McNaughton and Gunn, Ann Arbor, MI.
BOAG, P. т. 1983. The heritability of external morphology in Darwin's ground finches (Geospiza) on Isla Daphne Major, Galapagos. Evolution, 37, 877-894.

CHEVERUD, J. M. 1988. A comparison of genetic and phenotypic correlations. Evolution, 42, 958-968.

FALCONER, D. S. 1989. Introduction to Quantitative Genetics, 3rd edn. John Wiley and Sons, New York.

GRANT, P. R. 1983. Inheritance of size and shape in a population of Darwin's finches, Geospiza conirostris. Proc. $R$. Soc. B., 220, 219-236.

HARRISON, R. G. 1985. Barriers to gene exchange between closely related cricket species. II. Life cycle variation and temporal isolation. Evolution, 39, 244-259.

KLEIN, T. W., DEFRIES, J. C. AND FINKBEINER, C. T. 1973. Heritability and genetic correlation: standard error of estimates and sample size. Behav. Genet., 3, 355-364.

KOOTS, K. R. AND GIBSON, J. P. 1994. How precise are genetic correlation estimates? Proc. 5th World Cong. Gen. Appl. Livestock Prod., 18, 353-356. Department of Animal and Poultry Science, University of Guelph, Ontario, Canada.

MOUSSEAU, T. A. AND ROFF, D. A. 1987. Natural selection and the heritability of fitness components. Heredity, 59, 181-198.

MUELLER, L. D. 1979. A comparison of two methods for making statistical inferences on Nei's measure of genetic distance. Biometrics, 35, 757-763.

ROFF, D. A. 1986. The genetic basis of wing dimorphism in the sand cricket, Gryllus firmus and its relevance to the evolution of wing dimorphisms in insects. Heredity, 57, 221-231.

ROFF, D. A. 1990. Understanding the evolution of insect life cycles: the role of genetical analysis. In: Gilbert, F. (ed.) Genetics, Evolution and Coordination of Insect Life Cycles, pp. 5-27. Springer, New York.

ROFF, D. A. 1994. Evidence that the magnitude of the trade-off in a dichotomous trait is frequency-dependent. Evolution (in press).

ROFF, D. A. AND PREZIOSI, R. 1994. The estimation of the genetic correlation: the use of the jackknife. Heredity, 73, 544-548.

ROFF, D. A. AND MOUSSEAU, T. A. 1987. Quantitative genetics and fitness: lessons from Drosophila. Heredity, 58, 103-118.

SCHLUTER, D. AND SMITH, J. N. M. 1986. Genetic and phenotypic correlations in a natural population of song sparrows. Biol. J. Linn. Soc., 29, 23-36.

SIMONS, A. AND ROFF, D. A. 1994. The effect of environmental variability on the heritabilities of traits of a field cricket. Evolution (in press).

SIMONS, A. M. AND ROFF, D. A. 1995. The effect of environmental variability on the genetic correlation structure in a field cricket. Evolution (in press).

SOKAL, R. R. AND ROHLF, F. J. 1981. Biometry, 2nd edn. Freeman, San Francisco.

WILLIS, J. H., COYNE, J. A. AND KIRKPATRICK, M. 1991. Can one predict the evolution of quantitative characters without genetics? Evolution, 45, 441-444. 